\title{
Diálogo trinacional sobre el proyecto Bridge 47
}

\section{La Salete Coelho, Antonio Sianes, Talía Vela-Eiden}

La Salete (LS): Buenos días, soy La Salete Coelho, del equipo del proyecto Sinergias ED y estoy junto con Talía y Antonio para mantener un diálogo sobre el proyecto Bridge 471, con la de idea de compartir lo que está siendo esta experiencia entre nosotros en este partenariado ibérico. ¿ंNos presentamos para que quién no sepa quiénes somos?

Talía (T): Mi nombre es Talía Vela-Eiden, y trabajo como oficial para los partenariados de intercambio de conocimientos en $\mathrm{EADI}^{2}$, que es la asociación europea de institutos de investigación de desarrollo, y que es miembro del consorcio extenso de Bridge 47.

Antonio (A): Yo soy Antonio Sianes, profesor en la Universidad Loyola de Andalucía e investigador en la Fundación ETEA para el Desarrollo y la Cooperación, que es la institución que está haciendo la coordinación del partenariado ibérico, la red de colaboración entre universidades y ONGD portuguesas y españolas.

LS: Gracias. Talía, ¿̇podrás contarnos un poco cómo surgió el proyecto Bridge 47 y así presentarlo brevemente?

T: Bridge 47 surgió como una iniciativa de varias organizaciones de la sociedad civil europea, que, desde hace varias décadas ya, desde los 90 , han estado trabajando en el tema de educación para el desarrollo sostenible y educación para la ciudadanía y, ahora, educación para la ciudadanía global, lo que se denomina

\footnotetext{
${ }^{1}$ https://www.bridge47.org/

${ }^{2}$ European Association of Development Research and Training Institutes - https://www.eadi.org/
} 
global citizenship education. Hubo 3 o 4 proyectos anteriores a Bridge 47, que antecedieron esta iniciativa, y a partir de ahí es que se formó el consorcio de organizaciones que forman Bridge 47 cuyo objetivo principal es ir un paso más adelante y continuar las actividades de los proyectos anteriores. El consorcio de Bridge 47 reúne organizaciones y personas de la sociedad civil europea que trabajan en el tema y se enlazan con la comunidad europea y sus instituciones. El objetivo es difundir los objetivos de la educación para la ciudadanía global, desde Europa, ir construyendo puentes, por eso se llama Bridge, puentes de intercambio entre las organizaciones de la sociedad civil europea con académicos, instituciones empresariales, sindicatos, y otros y también explorar estas conexiones, estos puentes, entre Europa y otras regiones del mundo.

A: Yo recuerdo que cuando Talía y los colegas de EADI me comentaban el significado de Bridge, me sirvió mucho ponerme en antecedentes. Hay mucha gente, en España y Portugal que identifica muy bien esa red que era DEEEP ${ }^{3}$ y cuando ves Bridge 47 como una continuación de todo eso, con la idea de, como bien dice Talía, ampliar el alcance, a mí me aclaró mucho. Desde entonces siempre lo utilizo cuando estoy intentando enmarcar el proyecto. Es un proyecto que surge con ciertas novedades porque nace en el marco de la Agenda 2030, pero que bebe de todas esas décadas de trabajo.

LS: Yo también tuve la posibilidad de participar en encuentros internacionales de DEEEP y les debo mucho, pues fue mi primera oportunidad internacional en este ámbito de acción. Talía, Antonio ha mencionado la Agenda 2030. ¿Quieres hablarnos un poco sobre esta conexión con este contexto global?

T: El nombre del proyecto, el 47, se refiere al Objetivo del Desarrollo Sostenible (ODS) 4 y específicamente al denominado target, o sea, objetivo específico 4.7. Allí es donde, por primera vez, se hace referencia a la Educación para la Ciudadanía Global como objetivo, como uno de los conceptos para llevar a cabo la implementación, la puesta en ejecución del objetivo del desarrollo 4 que es educación. Esto fue definitivamente lo que lanzó el proyecto a hacer esas conexiones globales, a tratar también de involucrar a las organizaciones europeas con lo que está pasando a nivel de Naciones Unidas y con redes similares a Bridge, que existen, por ejemplo, en Asia. Juntos seguir buscando la implementación de este objetivo,

\footnotetext{
3 Proyecto implementado por el grupo de trabajo de Educación para el Desarrollo (Development Awareness Raising and Education Forum - DARE Forum) de CONCORD, Confederación de Organizaciones No Gubernamentales para el Desarrollo, durante 12 años (en 4 fases de 3 años cada una) y financiado por la Comisión Europea.
} 
el desarrollo sostenible a través del concepto de Educación para la Ciudadanía Global, sin olvidar el tema de Educación para el Desarrollo Sostenible.

LS: Sí, claro. ¡Es lo que da el nombre al marco!

T: También es importante tener en cuenta que el organismo de Naciones Unidas que está encargado de la implementación del objetivo 4 es UNESCO. Eso se olvida muchas veces en todo este contexto, y es importante recordarlo porque eso implica también que hay determinados marcos sobre cómo es que el objetivo va a ser medido, va a ser implementado, las contribuciones que se esperan y en qué foros es que esas contribuciones se van a llevar a cabo. La reunión anual del equipo de política de alto nivel de Nueva York y las reuniones que UNESCO organiza sobre el tema de la educación para el desarrollo sostenible son foros donde también Bridge 47 quiere incorporarse o ya se ha incorporado para poder llevar a cabo este trabajo de construcción de puentes y más enlaces entre las organizaciones de la sociedad civil y otros sectores.

A: Yo cuando leí la agenda, buscando evidentemente referencias al tema de la Educación para el Desarrollo y Ciudadanía Global (ED/ECG), a mí el 4.7 no me saltó directamente a la mirada, es decir, me costó reinterpretarlo para encontrar el hueco que yo considero deberían jugar las educaciones transformadoras en la Agenda. Al estar en el objetivo 4, y como dice Talía, es competencia de UNESCO, entonces claro, coge mucha importancia el sustantivo "educación" ... ¿ Cómo habéis visto vosotros, en Portugal, que se integra en la agenda 2030 todo este tema de ciudadanía?

LS: Yo no estoy muy involucrada en esta discusión, pero veo la Agenda mucho más como un marco total para la Educación para el Desarrollo y la Ciudadanía Global, dentro del cual puedes trabajar todas sus temáticas: las temáticas ambientales, el consumo responsable, el género, etc. Pero creo que ha sido importante ver el concepto de Ciudadanía Global reflejado en este documento, porque creo que faltaba un poco esta legitimación internacional. Lo que digo es que creo que la ciudadanía global está en todos los objetivos, pero es importante que haya esta meta, 4.7, que lo diga expresamente, que la ciudadanía global es algo a alcanzar, es positivo. ¡Claro que no se agota en el objetivo 4 de educación! Y sabes, la mirada de ciudadanía global es importante también para criticar los propios ODS, para cuestionar, por ejemplo, la utilización del concepto "crecimiento económico", que a lo mejor deberemos pensarlo ¿qué tipo de desarrollo queremos?

A: Sí, sin duda esa perspectiva de que toda la Agenda debe mirarse en clave de ciudadanía global ofrece un marco de trabajo mucho más sugerente. 
LS: Talía, los puentes son un instrumento para hacer colaboración. ¿Cuál es el papel de la colaboración en el proyecto?

T: La idea de Bridge era salir de esa burbuja de resonancia en que solamente se encontraban los mismos expertos y buscar fuera de este grupo aliados, organizaciones y personas que de algún modo encuentran sinergias, como el nombre de la red que ustedes tienen en Portugal. Cada uno de los miembros de Bridge tiene fortalezas en un sector respectivo - educación para adultos, educación no formal, educación en empresas privadas - dependiendo de los contextos en que las organizaciones han estado trabajando. La idea fue reforzar, hacer la red más sólida a través de Bridge.

\section{LS: ¿̨Y cómo surge la participación de EADI?}

T: En el caso de EADI la fortaleza es tener una red de académicos trabajando en el tema del desarrollo, y el tema educación para el desarrollo sostenible siempre ha estado presente de algún modo en las actividades, pero no de manera específica. En EADI no se tiene actualmente un grupo de investigación o un grupo de trabajo sobre educación para el desarrollo sostenible o ECG. Se da por sentado, como los académicos enseñan en el área de estudios de desarrollo que necesariamente ese tema está incorporado. Ello nos hace reflexionar sobre los métodos de enseñanza que utilizamos, los enfoques, las aproximaciones, etc.

También vimos que muchos de los académicos que son miembros de EADI ya tenían esta sinergia entre academia y sociedad civil. Estas relaciones, estas conexiones, ya existían. Entonces ¿̇por qué no aprovecharlas?

Así empieza a pensarse primero en elementos comunes para una colaboración, una agenda en común. No sabemos a dónde llegaremos con ella, pero definitivamente esta va a contribuir a solidificar o a hacer más visible el tema de educación para la ciudadanía global.

LS: Ha sido una oportunidad para hacer cosas juntos, ¿̇no?

T: Claro. EADI pretende facilitar ese proceso, por lo menos encaminarlo, para que los aliados e interesados piensen en términos concretos, ¿̇qué podemos hacer de acá a 3 años o a 5 años para contribuir a fortalecer el ODS 4.7? Como ustedes, en la red ibérica, que están haciendo cosas concretas, como la publicación o las conferencias en base a sus intereses temáticos. En otras regiones recién están empezando a construir una red, en otras la colaboración existía pero en otras instancias, con otros objetivos. Los contextos y los temas son distintos entre las redes. 
A: En España esa colaboración es, desde hace muchos años, muy asidua. Creo que en nuestro país tiene mucho que ver con el hecho de que, cuando se empieza a desarrollar el sistema de cooperación, donde nace en un principio el tema de $E D$, estamos saliendo de una dictadura en los años 70 . Entonces en los 80 se produce una explosión en todos los ejercicios de ciudadanía, explosión a la que se suma todo el mundo de manera muy convencida. Los académicos hacen calle, y las sociedades que están a pie de calle quieren incidir en la academia. De ese contexto surgen muchísimas entidades del tercer sector y ONG con un componente académico muy fuerte, como por ejemplo economistas sin fronteras, ingeniería sin fronteras, etc.

\section{LS: Ha sido un proceso muy dinámico que os distingue.}

T: Creo que eso es un elemento diferencial de esta red ibérica y quizá tenga un poco esos antecedentes históricos. Aquí hay una red de académicas y académicos que es REEDES4, que forma parte de EADI, y REEDES y la coordinadora española de ONG están en constante comunicación, es decir, somos miembros mutuos una red de la otra, tenemos voz e incidencia una y otra en las decisiones de cada institución. El trabajo es muy natural y muy rápidamente se articulan esas redes en las que casi todos los perfiles somos mixtos - yo a lo mejor soy más académico pero trabajo en una ONG, como es la fundación, que tiene proyectos de educación para el desarrollo y ciudadanía global. Yo creo que ese perfil mixto es una riqueza muy importante para un proyecto y una riqueza que ya se da en nuestro contexto. ¿¿ en Portugal?

LS: En Portugal la historia es distinta. Porque tuvimos la dictadura hasta el 74 y después ha habido un enfoque muy grande en la cooperación para el desarrollo, la ED/ECG no ha sido muy desarrollada en Portugal hasta muy recientemente. Por ejemplo, nuestra primera estrategia es de 2010. Nuestro grupo, Sinergias, ha empezado con un proyecto y ahora nos llamamos una comunidad de interesados e interesadas en estos temas, pero el proyecto ha nacido exactamente para hacer este puente entre la academia y la sociedad civil. En Portugal da ED ha entrado por la sociedad civil, no ha ido directamente a la universidad, y cuando ha ido ha sido por estos perfiles mixtos que hablamos, en Sinergias nos llamamos los híbridos, los que estamos en los dos lados. El proyecto Sinergias ha sido importante para poner en conjunto a estas personas y ha sido esto también que hemos compartido en este partenariado ibérico, un poco esta comunidad que ya estaba en marcha en Portugal desde 2013.

${ }^{4}$ Red Española de Estudios de Desarrollo - https://reedes.org/ 
A: Talía, y sobre este Partenariado de Intercambio de Conocimientos. ¿̇Qué es lo que os ha llevado a elegir las tres zonas geográficas de los partenariados?

T: En realidad también se inició el trabajo a partir de las propias redes de EADI-hay algunas más dentro de lo que es activismo, otras más en lo que es entrenamiento, pero también hay un grupo que está más interesado en estos intercambios académicos. Empezamos, entonces, por identificar un grupo de inicio, un grupo semilla de personas que querían incorporarse a esta iniciativa y empezar a construir lo que ustedes en Sinergias Portugal ya han logrado que fuera formar una comunidad de gente interesada en Europa sobre estos temas de ED/ECG. Entonces es así que se hacen memorandos de entendimiento, como, por ejemplo, con Fundación ETEA, que fue uno de los primeros, también con Mundu de Dinamarca, con Ambrela (la organización de ONG Eslovaquia), con Sloga (la organización de asociación de ONG de Eslovenia). Desde esa base se firmaron otros memorandos de entendimiento con la Universidad de Stirling (Escocia) y la Universidad Comenius en Bratislava (Eslovaquia).

LS: ¿̇Y lo que diferencia los partenariados de intercambio de conocimientos de otras redes que existen en el momento en Europa?

T: Me preguntan eso muchas veces. Yo pienso que en las otras redes también se está haciendo un trabajo importante - escribir libros, producir papers académicos, hacer conferencias académicas - pero nuestro objetivo no es ese, es pensar en cómo efectivamente los académicos pueden contribuir de manera realmente significativa a cambios en política a nivel europeo, nacional, europeo y ahora también global, a través de las redes de intercambio. Teníamos el plan inicial de llevar a algunos miembros del partenariado de conocimiento a la reunión en Nueva York de Naciones Unidas, para mover un poco esa discusión a otro nivel, que haya un diálogo en el que los académicos presenten evidencia, presenten el resultado de sus éxitos.

A: Como parte del proyecto y como parte de una de las redes, yo creo que la colaboración dentro de cada red es una cosa que ya se está consiguiendo. Pero donde yo veo el principal reto es que entre las redes tengamos capacidad de hacer aprendizaje, es decir, que seamos redes de intercambio, no solo dentro de la red sino entre redes.

Hay una cosa que siempre me ha llamado mucho la atención en España. Yo empecé a trabajar en este tema en el 2011 (relativamente reciente) y veía como que estábamos recorriendo un camino que a lo mejor era necesario recorrer, pero que 
ya en otros contextos los habían recorrido y se había generado mucha evidencia sobre qué funcionaba, qué no, los riesgos de la colaboración o las dificultades previsibles, etc. Por eso estoy convencido de que si estas redes pudieran compartir esos aprendizajes que se producen a velocidades distintas y en temáticas distintas sería lo ideal. Recuerdo cómo durante mi proceso formativo, conocer los casos de Japón o de Irlanda, sobre los cuales he leído mucho, me ayudó a dar algunos pasos más rápido. Leía esas experiencias y pensaba: "estamos replicando algo que en estos contextos ya se han planteado hace 10 ○ 20 años".

LS: Es verdad que podremos aprender con otras experiencias, pero también es verdad que, en educación, sabes que el camino de cada uno es el suyo y tiene que aprender haciéndolo muchas veces. Pero lo que queríamos también con Sinergias y con la revista era esto, tener una plataforma de experiencias compartidas en lenguas distintas (por eso nuestra idea de poder publicar en inglés, en español, en portugués) para compartir y para ver los caminos que ya han sido recorridos para que podamos aprender con ellos y no hacer lo mismo y no demorar tanto tiempo. Sin embargo, hay que reconocer que cada contexto es distinto, y hay cosas que se tienen que descubrir por sí mismas, leer es importante, nos abre un poco la mente, nos da pistas, pero...

\section{A: ¡Verdad!}

T: Yo creo, además, que es esa la razón por que la contribución de los académicos debe ser elevada en estos contextos de política global. Para poner en diálogo perspectivas de geografías distintas - en América Latina, la experiencia específica de todo lo que es educación adulta, movimientos sociales, y las líneas freirianas; en los países del norte, Irlanda, Escocia, se está ahora en esta discusión poscolonial, decolonial. A estos les va a interesar muchísimo escuchar vuestra experiencia que viene de lo que es lberoamérica, estas vuestras sinergias e intercambios con América Latina. El tema del poscolonialismo tiene muchos matices desde el británico, anglosajón, a los de Portugal y España. Teníamos el plan de tener un foro, ahora en La Haya, en junio, donde, por primera vez los participantes de las redes de intercambio se reunieran y empiecen a dialogar sobre esos matices que son importantes. También eso influye en cómo se entiende el tema de derechos humanos - una visión liberal, o una visión más cívica de los derechos humanos, o una visión más jerárquica. ¿ $\dot{\mathrm{Y}}^{Y}$ con los países que vivieron antes detrás de la cortina de hierro, de Europa del Este? Para ellos lo que es ECG tiene mucho que ver con temas de derechos cívicos, la lucha por la libertad de información, la libertad de expresión. Creo que esta es la posibilidad ofrecida por Bridge, de buscar esa conversación, ese intercambio, viendo esas perspectivas distintas. 
LS: Esto es un reto para el futuro del Bridge, sin duda, construir puentes entre las tres redes.

A: Ahí está el reto, es muy importante entender las prioridades, los tiempos, las asunciones que tiene cada actor, $y$ tener la sensibilidad para ver que son distintas dentro de cada red y a la vez son distintas entre las redes. Es importante ver cómo casamos todo eso de cara al futuro para que, efectivamente, alcancemos una colaboración que sea sostenible y que dé lugar a una red que aprenda, que genere sinergias, que tienda puentes, etc.

LS: Talia, sabemos que del evento de Helsinki ha resultado el documento Envision 47. ¿Quieres hablarnos un poco sobre él?

T: Este documento, Envision, presenta la visión, de cómo este concepto de educación para la ciudadanía global puede ser implementado, puesto en práctica en la realidad, primero, en Europa y, a partir de ahí, usando la influencia y el peso de Europa a nivel internacional que también se extienda a otras regiones a nivel global. Dentro de Bridge hay un componente de trabajo que se llama la red global que colecta las contribuciones de los diferentes miembros participantes de Bridge y los lleva a un nivel más alto. Este documento es una especie de hoja de ruta de cómo implementar, de cómo hacer realidad que los objetivos de la ECG sean incorporados en políticas a nivel europeo y mundial.

A: ¿̨Cómo fue el proceso de deliberación para llegar a una hoja de ruta compartida?

T: Eso fue muy interesante. Para elaborar este documento se reunieron muchas de las personas que habían estado participando en las redes de Bridge en los últimos diez años, incluyendo también los nuevos incorporados como los miembros de la Red Ibérica, y a través de una metodología participativa se llegó a elaborar un documento específico muy preciso con objetivos, con temas, actividades... Se trató de aterrizar muchas de las ideas que se tenían en algo específico que pueda ser efectivamente usado por los llamados decisores políticos o los tomadores de decisiones. Ese es el objetivo, el uso, la razón de ser del documento Envision 47.

LS: ¿Cuál es el rol de los Partenariados de Intercambio de Conocimiento en este documento?

T: Efectivamente necesitamos del aporte, de la contribución de los académicos de Europa y también expertos de otras regiones, en cada uno de los puntos identificados como prioritarios para la implementación de la educación para la ciudadanía global a nivel de políticas. Esa es la siguiente etapa hacia donde nosotros, 
EADI, deseamos llegar. ¿Cómo operacionalizar eso ya desde un punto de vista concreto, de recursos, de actividades y por supuesto de ideas concretas? Eso se hace con la red global y no tanto con las redes de conocimiento, pero las redes regionales continúan siendo el foco. Hay nuevos participantes incorporándose en las redes y quieren ahora ser parte porque ven que justamente es posible llegar a algo concreto y específico con el trabajo que Bridge estaba haciendo y creo que esa es una de las razones que también hace que sea atractivo, o sea, no solamente sentarse y discutir todo. Somos académicos, yo también enseño en la universidad, y me encanta discutir cosas teóricas, pero el hecho es que también llega un momento en que quieres intervenir en la práctica. Ese es justamente el punto que sigue siendo el objetivo a largo plazo de este grupo de intercambio de conocimiento. Por ejemplo, el tema de los indicadores - hay que trabajar en ellos. Puede ser que alguna de las redes diga, "estos indicadores nos interesan en el tema de intercambios intersectoriales", "tenemos ya experiencia con gobiernos locales, gobiernos regionales". Eso es como veo el proceso - identificar, en la hoja de ruta, cuáles son los temas en los que ustedes tiene las mayores fortalezas, y aportar contribuciones.

\section{LS: Esto es un reto para nosotros, Antonio.}

A: A mí esto me llama mucho la atención porque muchas de las cosas que dice Talía al final de una manera $u$ otra ya las estamos teniendo en cuenta, $\dot{2}^{\text {no? }}$ Por ejemplo, en el ciclo de webinars que estamos preparando, el webinar que vamos a hacer en torno al Bridge, va a ir precisamente de cómo aterrizar la cuestión de la ECG a nivel de un gobierno local. O sea, cómo se aterriza a nivel de un gobierno local el tratar la ciudadanía global en clave de agenda 2030. Me resulta muy interesante que estemos muy alineadas en todo lo que se está hablando y me parece que es un poco la tónica general. Ojalá que esos retos lleven a que el alineamiento sea cada vez mayor y efectivamente demos también ese paso de lo teórico a lo aplicado.

LS: Para la comunidad Sinergias es muy importante esto también del conocimiento, de su producción, validación y diseminación, con una mirada poscolonial. Que esta colaboración entre sociedad civil y academia también generen cuestiones, nuevas metodologías, nuevas formas de mirar el conocimiento. Estas discusiones son algo muy precioso para la comunidad Sinergias y creo que también por ahí vamos a aportar algo, sin duda porque hay algo en esta discusión que es muy fuerte.

T: Queda la invitación - mirad al documento de la hoja de ruta con este propósito de identificar vuestra contribución, para pensar en cosas específicas y concretas y continuar el diálogo con las otras redes. 
LS: Antonio, antes de terminar creo que podríamos hablar un poco sobre lo que hemos hecho entre nosotros, en la red ibérica. Estamos a punto de entrar en la parte final del camino, y es tiempo para hacer un balance.

A: Cierto. Si recuerdas, hace ya un año y medio, aprovechando que la fundación ETEA estaba organizando el congreso de REEDES en Córdoba, a finales del 18, nos sumamos al proyecto con el objetivo de intentar reforzar la conexión con Europa, que yo creo que es una cuestión muy importante tanto para España como para Portugal. No siempre sentimos esta conexión, y es muy importante sumarnos al proyecto europeo de ciudadanía global. La idea fue reunirnos en Córdoba. Invitamos a Vanessa Andreotti a dar una conferencia sobre cómo se estaba viendo todo este tema de ciudadanía global y de prácticas realmente transformadoras desde el punto de vista crítico, compartiendo una metodología que luego ha estado transversal en todo el Bridge. Si recuerdas, empezamos poquitas personas, básicamente de Portugal estabas tú, La Salete, en nombre de Sinergias, y de España estábamos algunas universidades y algunas ONG. El segundo encuentro que tuvimos en Galicia sí fue ya mucho más amplio. Creo que ya se vio una representación bastante mayor, sobre todo de colegas de Sinergias del Norte de Portugal, aprovechando su cercanía, y de España se sumaron colegas de Valladolid, de la propia Galicia, o de la Universidad de Barcelona. También estuvo Talía desde Bridge 47.

LS: En Portugal tenemos esta ventaja de tener una comunidad estructurada con muchas personas de la sociedad civil y de la academia, somos cerca de 40 ○ 50 personas que estamos en esta comunidad. Tenemos cuatro grupos, cuatro niveles, el equipo del proyecto - del Centro de Estudios Africanos de la Universidad de Oporto y de la Fundación Gonçalo da Silveira -, el grupo de la comunidad que está muy involucrado en esa tercera edición del proyecto, un grupo más global de personas que son amigas del proyecto, que reciben noticias de lo que pasa, pero que en este momento por alguna razón no pueden ser tan participativas, y el grupo de amigos y amigas internacionales - Alexandra Boni, por ejemplo, que ha participado en nuestras cosas desde el principio, personas del Global Education Network Europe ${ }^{5}$, del Academic Network of Global Education and Learning ${ }^{6}$, de América Latina, etc.

Cuando hemos recibido el contacto de Antonio para ir Córdoba, hemos pensado que para nosotros tenía sentido participar porque era una oportunidad para re-

\footnotetext{
${ }^{5}$ https://gene.eu/

${ }^{6}$ https://angel-network.net/
} 
forzar lazos que ya teníamos, para ampliarlos, estrecharlos y también aprovechar esta conexión ya con América Latina, porque lberia tiene sentido también con este pasado histórico.

T: ¿Queréis hablar de lo que estáis planeando?

LS: Teníamos muchas cosas, pero la situación actual nos ha hecho cambiar muchos planes. Nos íbamos a encontrar en Lisboa para el tercer encuentro de la red, aprovechando el encuentro internacional final de esta edición del proyecto Sinergias, pero lo hemos cambiado para hacer unas webinars, donde serán presentadas varias experiencias de colaboración y estamos preparando en conjunto un monográfico para la revista Sinergias - diálogos educativos para la transformación social y para la revista Fomento Social de la Universidad Loyola. Íbamos estar también en Canarias y en el encuentro final en La Haya. Pero creo que todos tenemos esta voluntad de continuar haciendo cosas porque creemos. No estamos aquí solamente por los proyectos, sino que estamos en esto porque creemos que es importante en este momento hablar de estas cosas, de trabajar, politizar un poco también esta intervención en ECG para hacer frente a los retos que el mundo nos coloca en este momento. 